Rev. Int. Contam. Ambie. 36 (4) 997-1010, 2020

https://doi.org/10.20937/RICA.53495

\title{
ALTERACIONES DEL CICLO MENSTRUAL DE ADOLESCENTES EXPUESTAS NO OCUPACIONALMENTE A UNA MEZCLA DE PLAGUICIDAS DE UNA ZONA AGRÍCOLA DE SAN LUIS POTOSÍ, MÉXICO. ESTUDIO PILOTO
}

Alterations of the menstrual cycle of young adults not occupationally exposed to a mixture of pesticides from an agricultural area of San Luis Potosí, Mexico. A pilot study

\section{Andrea RODRÍGUEZ-LÓPEZ ${ }^{1,2}$, Rebeca MEJÍA-SAUCEDO ${ }^{1}$, Jaqueline CALDERÓN-HERNÁNDEZ ${ }^{3}$, Vanessa LABRADA-MARTAGÓN ${ }^{4}$ y Leticia YÃNEZ-ESTRADA ${ }^{1 *}$}

${ }^{1}$ Laboratorio de Género, Salud y Ambiente, Facultad de Medicina, Universidad Autónoma de San Luis Potosí, Av. Venustiano Carranza 2405, Col. Loma Alta, 78210 San Luis Potosí, San Luis Potosí, México.

${ }^{2}$ Departamento de Físico-Matemáticas, Facultad de Ingeniería, Universidad Autónoma de San Luis Potosí. Dr. Manuel Nava 8, Col. Zona Universitaria Poniente, 78290 San Luis Potosí, San Luis Potosí, México.

${ }^{3}$ Centro de Investigación Aplicada en Ambiente y Salud (CIAAS), CIACYT-Facultad de Medicina, Universidad Autónoma de San Luis Potosí, Sierra Leona 550, Lomas 2a Sección, 78210 San Luis Potosí, San Luis Potosí, México.

${ }^{4}$ Facultad de Ciencias, Universidad Autónoma de San Luis Potosí, Av. Chapultepec 1570, Col. Privadas del Pedregal, 78295 San Luis Potosí, San Luis Potosí, México.

*Autora para correspondencia: lyanez@uaslp.mx

(Recibido: enero de 2019; aceptado: enero de 2020)

Palabras clave: plaguicidas organoclorados, plaguicidas organofosforados, hormonas sexuales femeninas, xenoestrógenos, exposición no ocupacional.

\section{RESUMEN}

Los contaminantes orgánicos persistentes son un grupo de compuestos químicos de preocupación mundial debido a los daños a la salud que ocasiona la exposición a los mismos. Es destacable su capacidad como perturbadores endocrinos, siendo las mujeres en edad reproductiva una de las poblaciones más vulneradas. El objetivo del presente estudio fue evaluar el efecto de la exposición a una mezcla de plaguicidas en el ciclo menstrual de adolescentes residentes en El Refugio, San Luis Potosí, México, comunidad cuya actividad preponderante es la agricultura. La población de estudio estuvo conformada por 29 adolescentes en un intervalo de edad 12 a 17 años, quienes fueron capacitadas para registrar en un diario/bitácora todos los eventos relacionados con su ciclo menstrual. Se midieron los niveles séricos de las hormonas sexuales por quimioluminiscencia, así como las concentraciones plasmáticas de los plaguicidas organoclorados (OC) y los niveles urinarios de los metabolitos dialquilfosfatos (DAP) de los agroquímicos organofosforados mediante cromatografía de gases acoplada a espectrofotometría de masas. El $41.6 \%$ de las participantes tuvo alguna alteración en los niveles de la hormona folículo estimulante y de progesterona. De éstas, el $62 \%$ presentó plaguicidas OC en plasma ( $\Sigma \mathrm{OC}=1651.25 \mathrm{ng} / \mathrm{g}$ de lípidos) y el $66 \%$ tuvo niveles detectables de DAP ( $\Sigma \mathrm{DAP}=153.82 \mathrm{mg} / \mathrm{g}$ de creatinina). Estos resultados reflejan daño endocrino que pudiera estar asociado a la exposición a una mezcla de plaguicidas organoclorados y organofosforados. 
Key words: organochlorine pesticides, organophosphate pesticides, female sex hormones, xenoestrogens, nonoccupational exposure.

\begin{abstract}
Persistent organic pollutants are a group of chemical compounds of global concern due to the health damages caused by the exposure to them. Their capacity as endocrine disrupters is one of their main characteristics, being women in reproductive age the most vulnerable population. The aim of the present study was to evaluate the effect of exposure to a mixture of pesticides in the menstrual cycle of a sample of 29 teenagers between the ages of 12 and 17 years old from El Refugio, San Luis Potosi, Mexico, a community whose predominant activity is agriculture. The participants were trained to record in a diary/ logbook all the events related to their menstrual cycle. Serum levels of sex hormones were measured by chemiluminescence, and plasma concentrations of organochlorine (OC) pesticides and the level of dialkyl phosphates metabolites (DAPs) of organophosphorus agrochemicals presented in urine were measured through gas chromatographymass spectrometry. Around $41.6 \%$ of the participants presented some alteration in their hormonal levels, mainly of follicle-stimulating hormone and progesterone. Of these, OC pesticides in plasma were detected in $62 \%(\Sigma \mathrm{OC}=1651.25 \mathrm{ng} / \mathrm{g}$ of lipid $)$, and 66 $\%$ had detectable levels of DAPs ( $\Sigma \mathrm{DAP}=153.82 \mathrm{mg} / \mathrm{g}$ of creatinine). Together, these results suggest damage to the endocrine system that could be generated by exposure to a mixture of OC and organophosphorus pesticides could generate.
\end{abstract}

\section{INTRODUCCIÓN}

El ciclo menstrual $(\mathrm{CM})$ es un evento biológico que está regulado hormonalmente y puede verse afectado por diversos factores, entre ellos una mala alimentación, extrema actividad física, estrés, edad, y consumo de alcohol y tabaco, entre otros (Roselló et al. 2003, Zellnera et al. 2004, Escobar et al. 2010, Wojtys et al. 2014). Un factor adicional que puede contribuir a alterar el CM es la exposición a plaguicidas (Farr et al. 2004, Bretveld et al. 2006, Fowler et al. 2012). Los reportes en la literatura muestran que la exposición a compuestos organoclorados (OC) fueron los primeros eventos con los que se observó esta asociación (Bhatt 2000, Charboneau y Koger 2008, Germaine et al. 2011). Modelos animales de exposición a hexaclorobenceno mostraron diversas alteraciones como cambios en la duración del CM, reducción de los niveles séricos de estradiol, así como la generación de ciclos anovulatorios (Tiemann 2008). El dicloro difenil tricloroetano (DDT) y su metabolito dicloro difenil dicloroetileno (DDE) fueron los principales plaguicidas denominados perturbadores endocrinos (xenoestrógenos), ya que poseen propiedades estrogénicas y pueden afectar al sistema reproductor (Uzumcu y Zachow 2007). Dichos compuestos pueden tener efectos directos o indirectos en la estructura del receptor hormonal, tales como alterar su función, cambiar su síntesis o su afinidad por moléculas específicas, y/o modular el número de receptores (Roselli et al. 2000, Nicolopou y Pitsos 2001, Tiemann 2008, Melmed et al. 2017). Widham et al. (2005), observaron en mujeres asiáticas inmigrantes una asociación entre las concentraciones en suero de 4,4'-DDE y la presencia de ciclos menstruales cortos, atribuyendo a este compuesto químico la reducción de la fase lútea del CM. También reportaron una asociación positiva entre las concentraciones plasmáticas de 4,4'-DDE y niveles séricos de progesterona y estradiol alterados (Windham et al. 2005).

En otro estudio realizado en Iowa y Carolina del Norte, en el que a lo largo de un año se entrevistó a 3500 mujeres que residían o trabajaban en campos agrícolas, se obtuvo información sobre las características de su CM. Los autores señalaron que la exposición a diferentes mezclas de plaguicidas como atrazina, lindano, malatión y carbamatos, entre otros, afectó la duración tanto de los ciclos como de los periodos menstruales, generando sangrados intermenstruales, así como dificultad para el embarazo (Farr et al. 2004). Aunado a lo anterior, se ha reportado que la exposición a compuestos clorados puede afectar la edad de inicio de la menarquia, así como la duración de los periodos (Ouyang et al. 2005). 
Respecto de los plaguicidas organofosforados, no existen datos en la literatura sobre estudios en poblaciones humanas en los que se evalúe la asociación entre exposición a estos agroquímicos y alteraciones en los niveles de hormonas sexuales. Los estudios en modelos animales también son escasos. En uno de éstos, realizado en ratones Peromyscus leucopus noveboracensis a los cuales se expuso a 50 y $100 \mathrm{mg} / \mathrm{kg}$ de atrazina, respectivamente, se registró, además de una importante inhibición de la acetilcolinesterasa, una reducción del $29 \%$ de la concentración basal de la hormona luteinizante (LH) (Foradori et al. 2011). En otro estudio realizado en codornices que fueron expuestas durante 10 días por vía oral a diferentes concentraciones de paratión adicionado a su alimento, se observó que éste detenía el desarrollo folicular generando ciclos anovulatorios y una reducción de la concentración sérica de LH (Rattner et al. 1982). De igual manera, son escasos los reportes en la literatura relacionados con la probable asociación entre exposición a agroquímicos organofosforados y alteraciones en el CM de mujeres mexicanas. Un estudio de casos y controles en mujeres adultas del estado de Chihuahua encontró una relación entre la exposición a una mezcla de plaguicidas (malatión, paratión, clorpirifós y dimetoato) y el retraso en el crecimiento intrauterino (Levario et al. 2004).

Una importante limitante de los estudios previos en mujeres es que tanto la evaluación de la magnitud de la exposición a los agroquímicos, como el registro del comportamiento del ciclo menstrual, se verificaron con base en la aplicación de cuestionarios sobre usos y costumbres, así como de entrevistas. De ahí la relevancia del presente estudio, cuyo objetivo fue evaluar en adolescentes residentes en una zona agrícola de San Luis Potosí, México, la exposición a plaguicidas organoclorados y organofosforados mediante el monitoreo de sus concentraciones en plasma y de los niveles urinarios de metabolitos DAP, respectivamente. Asimismo, se valoró la posible asociación de dicha exposición con alteraciones en las características del ciclo y del periodo menstrual, con base en el registro en un diario de eventos ocurridos a lo largo de éstos y los niveles séricos de las hormonas sexuales.

\section{MATERIALES Y MÉTODOS}

\section{Sitio de estudio}

El estudio se realizó en El Refugio, Ciudad Fernández, el cual se localiza a $133 \mathrm{~km}$ al este de la ciudad de San Luis Potosí (Fig. 1). Su principal actividad económica es la agricultura (maíz, frijol, cítricos y chile, entre otros) (INAFED-SEGOB 2010). Estudios previos realizados por nuestro grupo de investigación en esta misma área, han documentado el uso histórico de plaguicidas organofosforados (OF), OC y carbamatos, demostrando la exposición a mezclas de estos contaminantes químicos en población infantil (Yáñez 2000, Rivero 2012 y Ramírez-Jiménez et al. 2014).

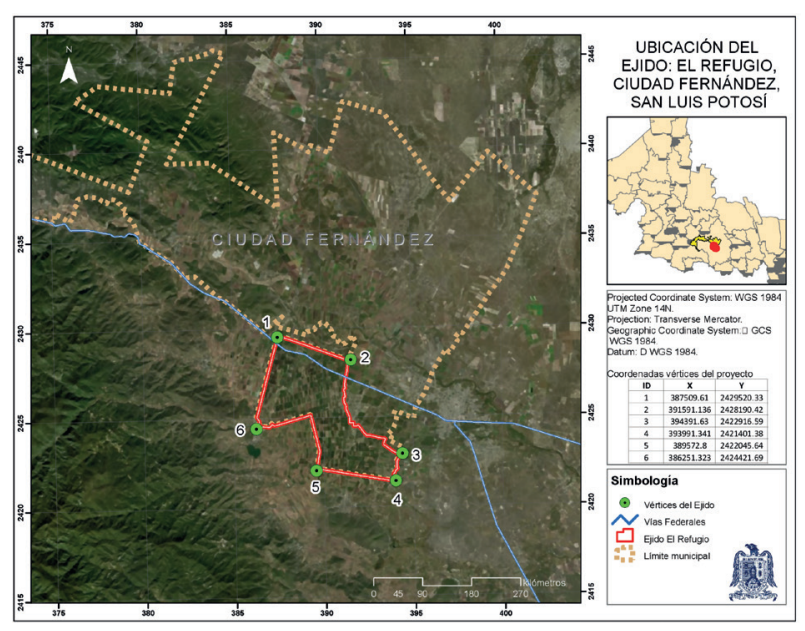

Fig. 1. Ubicación de la comunidad de estudio: El Refugio, Ciudad Fernández, San Luis Potosí. Fecha de elaboración: diciembre de 2014.

\section{Población de estudio}

Este estudio fue aprobado por el Comité de Bioética e Investigación de la Facultad de Medicina de la Universidad Autónoma de San Luis Potosí.

La muestra de adolescentes participantes se obtuvo de la base de datos de los estudios previos realizados en esta región por nuestro grupo de investigación (Yáñez 2000, Rivero 2012 y Ramírez-Jiménez 2014). Se les convocó a una reunión informativa con sus padres para explicarles el objetivo del estudio, los beneficios que recibirían, que éste era gratuito, que su participación era voluntaria y que podrían retirarse del mismo cuando así lo consideraran pertinente sin dar explicación alguna. Aquellas que aceptaron cooperar y cumplían con los criterios de inclusión, lo hicieron mediante la firma del consentimiento informado por parte de sus padres y el de asentimiento de cada una. Accedieron a participar en el estudio 32 adolescentes. Los criterios de inclusión fueron: edad de 12 a 17 años, residencia en la zona de estudio desde el nacimiento, menarquia mínima de un año previo al inicio del presente estudio (agosto de 2013), mujeres clínicamente sanas de acuerdo con el reporte de los padres. Los criterios de 
no inclusión fueron: estar embarazada y el uso de algún tipo de anticonceptivo hormonal. Los criterios de eliminación fueron: imposibilidad de recolectar alguna de las muestras biológicas, no registrar puntualmente todos los eventos relacionados con su ciclo menstrual en el diario/bitácora, embarazarse y no presentarse los días citados en el centro de salud para la evaluación correspondiente.

\section{Información general y usos y costumbres}

Se aplicó un cuestionario para registrar información de las participantes relacionada con su estado de salud en general y ginecológica, actividades extraescolares, actividad sexual, datos sobre su alimentación y actividades relacionadas con la exposición a plaguicidas, tales como su participación en labores en el campo agrícola. Se obtuvo el peso y la talla de cada una de las participantes para calcular su índice de masa corporal (CDC 2015), actividad realizada por personal capacitado para ello.

\section{Diario/bitácora (reporte del comportamiento del ciclo menstrual)}

Antes de iniciar el estudio se impartió una charla para explicar a las participantes el llenado correcto del diario/bitácora en el cual debían anotar información relacionada con su ciclo menstrual, desde el primero hasta el último día del periodo, la cantidad de flujo (número de toallas sanitarias empleadas por día) y los síntomas tanto premenstruales como menstruales, así como la presencia de dolor e incremento o disminución del apetito. Dicho registro se realizó durante 15 meses. A lo largo de este periodo se realizaron cuatro visitas sin previo aviso para verificar la anotación adecuada de la información.

Se consideró un ciclo normal aquel que comprendía de 24 a 31 días hasta llegar al nuevo periodo menstrual. En cuanto a la duración del periodo, se consideró normal aquel con un sangrado de tres a siete días. Cualquier variación del ciclo o del periodo que no estuviera dentro de estos intervalos se consideró como una alteración (Escobar et al. 2010). Respecto a la valoración del perfil hormonal, fue crucial la información registrada en el diario/bitácora, ya que esto permitió identificar en qué fase del CM se encontraban las participantes el día de la toma de la muestra de sangre; por ende, se facilitó la detección de niveles fuera del intervalo normal de las hormonas.

\section{Muestreo biológico}

Se recolectaron muestras de sangre y orina para las determinaciones analíticas. Las muestras de sangre las obtuvo personal calificado en ayunas por punción venosa, empleando material nuevo y estéril. Se recolectaron $5 \mathrm{~mL}$ en un tubo vacutainer sin anticoagulante para la determinación del perfil hormonal y $6 \mathrm{~mL}$ en un tubo vacutainer con heparina sódica para cuantificar las concentraciones plasmáticas de los plaguicidas OC. Para la cuantificación de los metabolitos dialquilfosfato (DAP) y los niveles de creatinina, se recolectó la primera orina de la mañana de cada participante durante siete días consecutivos en frascos de plástico, de boca ancha y estériles. Posteriormente se generó una muestra compuesta para su posterior análisis.

Las muestras se colocaron en una hielera con geles congelados para mantenerlas protegidas de la luz y entre 4 y $8{ }^{\circ} \mathrm{C}$ durante su traslado al Laboratorio de Género, Salud y Ambiente de la Facultad de Medicina de la Universidad Autónoma de San Luis Potosí (UASLP), ubicado a $2 \mathrm{~h}$ del sitio de estudio, donde se almacenaron a $-80{ }^{\circ} \mathrm{C}$ hasta su análisis.

\section{Cuantificación de los niveles séricos de hormonas sexuales}

Se determinaron los niveles séricos de $\mathrm{LH}$, de la hormona folículo estimulante (FSH), progesterona, estradiol y prolactina mediante un ensayo inmunométrico con dos sitios de unión quimioluminiscente en fase sólida, utilizando el equipo IMMULATE 1000. Este análisis se realizó en el Laboratorio de Medicina Nuclear de la Facultad de Medicina de la UASLP.

\section{Cuantificación de los niveles plasmáticos de pla- guicidas $\mathrm{OC}$}

Se empleó el método reportado por Hovander et al. (2000). Las muestras de plasma se descongelaron a temperatura ambiente. Antes de su análisis cromatográfico se les adicionó PCB141-C $\mathrm{C}^{13}$ como estándar interno a una concentración final de $10 \mathrm{ng} / \mathrm{mL}$ y se conservaron en refrigeración por $12 \mathrm{~h}$. Después de este tiempo, se realizó una desproteinización utilizando alcohol desnaturalizado y sulfato de amonio saturado. Para disminuir el efecto de matriz, se realizó una limpieza con dos columnas de Florisil en serie (activadas previamente con acetona y cloruro de metileno). Los analitos se eluyeron con una mezcla de hexano:cloruro de metileno (70:30), el extracto obtenido se concentró bajo corriente de nitrógeno hasta $100 \mu \mathrm{L}$ para su posterior análisis por cromatografia de gases-masas (CG-MS). Se cuantificaron los siguientes plaguicidas: a-hexaclorociclohexano (a-BHC), b-hexaclorociclohexano (b-BHC), g-hexacloriciclohexano (g-BHC), hexaclorobenceno, heptacloro, aldrin, cis-nonaclor, trans-nonaclor, 2,4'-dicloro difenil tricloroetano (2,4'-DDT), 4,4'-di- 
cloro difenil tricloroetano (4,4'-DDT), 2,4'-dicloro difenil dicloroetileno (2,4'-DDE) y 4,4'-dicloro difenil dicloroetileno (4,4'-DDE). Las concentraciones de plaguicidas $\mathrm{OC}$ se expresan por gramo de lípidos, los cuales se determinaron mediante un método colorimétrico empleando el ensayo comercial SPINREACT(R), de acuerdo con las especificaciones del comerciante. Las concentraciones plasmáticas de los plaguicidas OC están expresadas como mediana y percentiles y ajustadas por gramo de lípidos.

\section{Cuantificación de los metabolitos DAP en orina}

El método empleado fue el desarrollado por Valcke et al. (2006), con algunas modificaciones implementadas por Ramírez-Jiménez et al. (2014). Las muestras de orina se descongelaron a temperatura ambiente y antes de su análisis se les adicionó el dietil tiofosfato deuterado (DETP-d10) como estándar interno. Posteriormente se realizó una derivatización adicionando una mezcla de acetonitrilo, carbonato de potasio y bromuro de pentafluorobencilo. Los ésteres obtenidos fueron extraídos con una mezcla de cloruro de metileno al $8 \%$ en hexano; el extracto se evaporó bajo corriente de nitrógeno a $25^{\circ} \mathrm{C}$ hasta un volumen final de $100 \mu \mathrm{L}$ y se transfirió a un vial cromatográfico para su posterior inyección en el sistema CG-MS.

Los metabolitos cuantificados fueron: dimetilfosfato (DMP), dimetiltiofosfato (DMTP), dimetilditiofosfato (DMDTP), dietilfosfato (DEP), dietiltiofosfato (DETP) y dietilditiofosfato (DEDTP). Las concentraciones urinarias de DAP están expresadas como mediana y percentiles y ajustadas por gramo de creatinina.

La identificación de los plaguicidas OC y de los metabolitos DAP se realizó mediante el método de ion selectivo, mientras que para la cuantificación se utilizaron curvas de calibración. Este proceso analítico se realizó en un equipo de cromatografía de gases Agilent 7820A GC System acoplado a un detector de espectrometría de masas 5975 y un automuestreador 6514 A. Para el control de calidad se adicionaron concentraciones conocidas de los diferentes estándares certificados de los plaguicidas antes mencionados a las muestras blanco de plasma y orina, y éstas fueron procesadas en las condiciones previamente descritas. Se realizó la validación del método cromatográfico según la guía $\mathrm{AOAC}$, cumpliendo con todos los parámetros establecidos (repetibilidad, reproducibilidad, precisión, linealidad, porciento de recobro, límite de detección y límite de cuantificación) (AOAC 2015).

\section{Análisis estadístico}

Se realizó un análisis exploratorio de los datos para determinar su tipo de distribución mediante el método de Kolmogorov-Smirnov, encontrándose que no seguían una distribución normal, por lo que fueron transformados a logaritmo natural. Aún así no se ajustaron a una distribución normal, por lo que se empleó estadística no paramétrica para realizar las comparaciones respectivas. Se utilizó la prueba $U$ de Mann-Whitney para establcer si había alguna relación entre las concentraciones plasmáticas de los plaguicidas OC y urinarias de los DAP y el comportamiento del CM y el perfil hormonal.

Con la finalidad de determinar el grado de contribución de los agroquímicos a las alteraciones de las hormonas sexuales, se realizó un análisis discriminante para evaluar el nivel de significancia en la separación de los tres grupos de estudio conformados a priori (alterado, no alterado y en periodo menstrual), de acuerdo con las concentraciones hormonales de las jóvenes participantes. En el análisis se incluyeron los 12 plaguicidas OC y los seis metabolitos DAP que se analizaron como variables explicativas, las cuales fueron introducidas en el modelo utilizando el método estándar. Se emplearon el estadístico lambda de Wilks y las distancias de Mahalabis como estadísticos de bondad de ajuste considerando un nivel de significancia de $\alpha=0.05$ (Tabachnick y Fidell 1966). Los análisis estadísticos se realizaron con los paquetes SPSS v. 18 (SPSS 2009) y Statistica v. 8 (StatSoft 2009).

\section{RESULTADOS}

La población de estudio quedó conformada por 29 adolescentes (dos de las 32 jóvenes que habían aceptado participar en el protocolo fueron descartadas por embarazo y una tercera decidió no continuar). $\mathrm{La}$ edad media de las participantes fue de $14 \pm 2.05$ años. El $43.7 \%$ tiene huertos en su casa, de las cuales el $42.87 \%$ ayuda en las labores agrícolas y un $64.28 \%$ usa plaguicidas. E1 $75 \%$ de las adolescentes participantes pertenece a familias de jornaleros agrícolas.

En la figura 2 se muestra el número de $\mathrm{CM}$ que presentó cada una de las adolescentes a lo largo de los 15 meses que estuvo vigente el estudio. Se puede observar que algunas de las participantes identifican periodos importantes de amenorrea, ya que sólo tuvieron de cuatro a seis $\mathrm{CM}$, mientras que otras reportaron de 12 a 15 ciclos. Respecto al comportamiento del ciclo menstrual, el $56.26 \%$ de las adolescentes presentó un CM irregular, ya sea que éste fuese corto (menor a 24 días), o largo (mayor a 32 días). Con relación al periodo, el $24 \%$ de las adolescentes indicó haber tenido sangrados irregulares, 


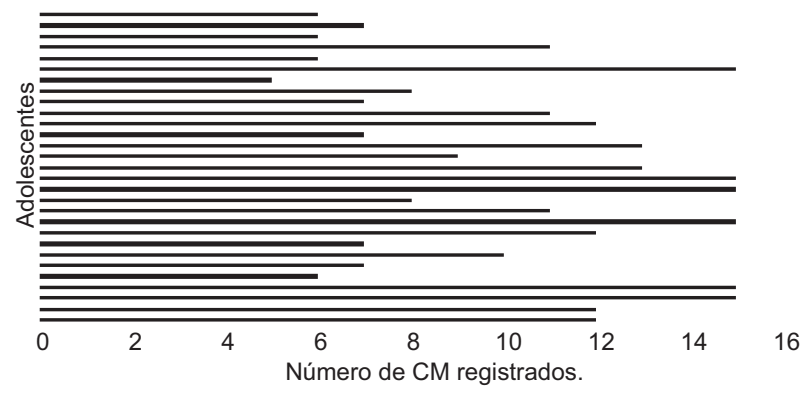

Fig. 2. Número de ciclos menstruales reportados a lo largo de 15 meses. Cada línea horizontal representa a una adolescente $(n=29)$.

ya sea que éstos fueran cortos (menores a cuatro días) o largos (mayores a siete días). Con relación a los niveles séricos de las hormonas sexuales, más del 40 $\%$ presentó alteraciones en su perfil hormonal, siendo la FSH la menos alterada, mientras que el $33 \%$ de las adolescentes presentó niveles de prolactina fuera del intervalo de referencia (de 0.05 a $0.4 \mathrm{ng} / \mathrm{mL}$ ).

Los métodos cromatográficos empleados para cuantificar los niveles plasmáticos de plaguicidas OC y urinarios de metabolitos DAP fueron previamente estandarizados y validados por nuestro grupo de investigación de acuerdo con los lineamientos de la AOAC (Yáñez 2000, Rivero 2012 y RamírezJiménez 2014, AOAC 2015), cumpliendo todos los parámetros establecidos, por lo que para el presente estudio sólo se validó la repetibilidad y la reproducibilidad. Ambos parámetros cumplen con los lineamientos de esta guía (por ciento del coeficiente de variación $[\% \mathrm{CV}]<15 \%$, datos no incluidos). Respecto del porcentaje de recobro, éste varió del 93 al $120 \%$ dependiendo del analito. También se calcularon los límites de detección y de cuantificación de cada uno de ellos. Por cada lote de ocho muestras se incluyeron dos muestras denominadas controles de calidad, una de concentración baja (CCB) y otra de concentración alta (CCA). Estas muestras se prepararon en el laboratorio adicionando concentraciones conocidas de cada uno de los plaguicidas de interés a una muestra de plasma u orina blanco, según fuese el caso. El resultado de la muestra problema sólo fue aceptado si las concentraciones estimadas de los CCB y CCA estaban dentro de la media $\pm 2 \mathrm{DE}$. Ningún lote fue rechazado.

En cuanto a la evaluación de la exposición a la mezcla de plaguicidas $\mathrm{OC}$ y $\mathrm{OF}$, el $96.9 \%$ de las adolescentes presentaron niveles detectables en plasma de g-BHC y urinarios de DEDTP, mientras que en el $93.7 \%, 87.5 \%$ y $84.2 \%$ de las participantes se detectaron DMTP, 4,4'-DDE y Cis-nonaclor, respectivamente. El compuesto con la concentración plasmática más elevada fue el 4,4'-DDE, seguido por el g-BHC y el 2,4'-DDE. La sumatoria de las concentraciones plasmáticas de los 12 plaguicidas OC estuvo en un intervalo de 356.10 a 13071.94 ng/g de lípidos, con una mediana de $1651.25 \mathrm{ng} / \mathrm{g}$ de lípidos. En cuanto a los percentiles se puede observar que el $75 \%$ de la población presentó concentraciones de 4,4'-DDE por debajo o iguales a $591.19 \mathrm{ng} / \mathrm{g}$ de lípidos y el $50 \%$ tuvo concentraciones de g-BHC menores a $304.60 \mathrm{ng} / \mathrm{g}$ de lípidos (Cuadro I). Con relación a los DAP, el metabolito que se detectó en concentraciones más elevadas fue el DMTP, seguido por el DEDTP y finalmente el DETP. El intervalo de concentración de la sumatoria de los seis DAP en las muestras de orina fue de 64.69 a $476.60 \mathrm{mg} / \mathrm{g}$ de creatinina, con una mediana de $153.82 \mathrm{mg} / \mathrm{g}$ de creatinina. En cuanto a los percentiles se observa que el $75 \%$ de la población presentó concentraciones de DEP menores a $11.79 \mathrm{mg} / \mathrm{g}$ de creatinina y el $50 \%$ tuvo concentraciones de DMTP por debajo de 5.30 $\mathrm{mg} / \mathrm{g}$ de creatinina (Cuadro II).

Al evaluar el efecto de la exposición a la mezcla de plaguicidas OC sobre la duración del CM dividido en tres grupos (corto, normal y largo) no se encontraron diferencias estadísticamente significativas, probablemente por el tamaño de la muestra, por lo que se realizó un segundo análisis conformado sólo por dos grupos: alterado (CM corto o CM largo) o normal. En la figura 3 se puede apreciar que aquellas adolescentes que presentaron alteraciones en el CM tienen concentraciones estadísticamente superiores del plaguicida 2,4'-DDE (317.76 ng/g de lípidos) en comparación con aquellas cuyo CM fue normal $(110.71 \mathrm{ng} / \mathrm{g}$ de lípidos $)(\mathrm{p}=0.03)$.

Respecto a la exposición a agroquímicos $\mathrm{OF}$ y la duración del CM, los niveles urinarios de DMTP en cuatro adolescentes que indicaron tener ciclos menores a 24 días (ciclo corto) fueron hasta tres veces mayores que en 13 participantes que reportaron tener ciclos normales ( 24 a 32 días) $(12.08 \mathrm{mg} / \mathrm{g}$ de creatinina vs $4.98 \mathrm{mg} / \mathrm{g}$ de creatinina, $\mathrm{p}=0.02$ ); sin embargo, estos resultados deben tomarse con cautela, dado al número tan pequeño de participantes que mencionaron tener CM cortos.

En cuanto a la duración del periodo, no se observaron diferencias significativas cuando se comparó su comportamiento con las concentraciones plasmáticas de plaguicidas OC, ni urinarias de los metabolitos DAP.

En relación con la comparación de concentraciones plasmáticas de plaguicidas OC y de las urinarias de metabolitos DAP con los niveles séricos de 
CUADRO I. CONCENTRACIONES PLASMÁTICAS DE LOS PLAGUICIDAS ORGANOCLORADOS.

\begin{tabular}{|c|c|c|c|c|}
\hline Plaguicida & $\begin{array}{c}\text { Concentración } \\
\text { (ng/g de lípidos)* }\end{array}$ & $\begin{array}{l}\text { Percentil } \\
25^{* *}\end{array}$ & $\begin{array}{l}\text { Percentil } \\
50^{* *}\end{array}$ & $\begin{array}{c}\text { Percentil } \\
75^{* *}\end{array}$ \\
\hline Aldrin & $\begin{array}{c}0.29 \\
(0.21-217.10)\end{array}$ & 0.25 & 0.29 & 0.34 \\
\hline $\mathrm{a}-\mathrm{BHC}$ & $\begin{array}{c}0.30 \\
(0.21-519.75)\end{array}$ & 0.24 & 0.30 & 0.40 \\
\hline $\mathrm{b}-\mathrm{BHC}$ & $\begin{array}{c}114.82 \\
(0.21-547.27)\end{array}$ & 0.26 & 114.82 & 201.79 \\
\hline g- BHC & $\begin{array}{c}304.60 \\
(0.41-1,190.16)\end{array}$ & 173.70 & 304.60 & 480.06 \\
\hline Cis-nonaclor & $\begin{array}{c}69.99 \\
(0.23-248.58)\end{array}$ & 50.56 & 69.99 & 91.32 \\
\hline $\mathrm{HCB}$ & $\begin{array}{c}0.58 \\
(0.21-72.92)\end{array}$ & 0.27 & 0.58 & 40.60 \\
\hline Heptacloro epóxido & $\begin{array}{c}0.33 \\
(0.21-271.03)\end{array}$ & 0.27 & 0.33 & 38.09 \\
\hline Trans-nonaclor & $\begin{array}{c}56.03 \\
(0.23-165.72)\end{array}$ & 45.18 & 56.03 & 70.32 \\
\hline 2,4'-DDE & $\begin{array}{c}259.14 \\
(0.21-826.88)\end{array}$ & 0.27 & 259.14 & 428.61 \\
\hline 4,4'-DDE & $\begin{array}{c}341.53 \\
(\mathrm{ND}-2,011.22)\end{array}$ & 165.32 & 341.53 & 591.19 \\
\hline $2,4^{\prime}$-DDT & $\begin{array}{c}0.29 \\
(0.21-149)\end{array}$ & 0.25 & 0.29 & 0.49 \\
\hline 4,4'-DDT & $\begin{array}{c}0.27 \\
(\mathrm{ND}-116.78)\end{array}$ & 0.24 & 0.27 & 0.34 \\
\hline$\sum \mathrm{OC}^{* * *}$ & $\begin{array}{c}1,651.25 \\
(356.10- \\
13071.94)\end{array}$ & 630.76 & 1651.25 & 7149.80 \\
\hline
\end{tabular}

*Los datos se reportan como mediana y entre paréntesis se ofrece las concentraciones mínima y máxima $(\mathrm{n}=29)$; **los percentiles fueron calculados con el paquete SPSS; *** OOC: sumatoria de la concentración plasmática de los 12 plaguicidas organoclorados (OC), expresada como la mediana y entre paréntesis los valores mínimo y máximo.

a-BHC: a-hexaclorociclohexano; b-BHC: b-hexaclorociclohexano; g-BHC: ghexacloriciclohexano; HCB: hexaclorobenceno; 2,4'-DDE: 2,4'-dicloro difenil dicloroetileno; 4,4'-DDE: 4,4'- dicloro difenil dicloroetileno; 2,4'-DDT=2,4'-dicloro difenil tricloroetano; 4,4'-DDT: 4,4'-dicloro difenil tricloroetano; ND: no detectable.

hormonas sexuales, es importante mencionar que el número de adolescentes con niveles alterados de una o más hormonas fue tan pequeño (progesterona: 2; estradiol: 3 ; LH: 3 ) que no se tienen los elementos estadísticos suficientes para considerar que la distribución de los niveles de exposición a los plaguicidas es diferente entre aquellas que presentaron alguna alteración en su perfil hormonal y las que no. Sin embargo, estos resultados pueden tener relevancia clínica y por ello no deben subestimarse.

La separación de los tres grupos de estudio conformados a priori de acuerdo con las alteraciones 
CUADRO II. CONCENTRACIONES URINARIAS DE LOS METABOLITOS DIALQUILFOSFATOS.

\begin{tabular}{lcccc}
\hline $\begin{array}{l}\text { Metabolito } \\
\text { DAP }\end{array}$ & $\begin{array}{c}\text { Concentración } \\
\text { (mg/g de creatinina) }\end{array}$ & $\begin{array}{c}\text { Percentil } \\
25^{* *}\end{array}$ & $\begin{array}{c}\text { Percentil } \\
50^{* *}\end{array}$ & $\begin{array}{c}\text { Percentil } \\
75^{* *}\end{array}$ \\
\hline DEP & $\begin{array}{c}0.001 \\
\text { (ND-175.27) }\end{array}$ & 0.001 & 0.001 & 11.79 \\
\hline DETP & $\begin{array}{c}1.08 \\
(\mathrm{ND}-21.23)\end{array}$ & 0.001 & 1.08 & 3.37 \\
\hline DEDTP & $\begin{array}{c}1.25 \\
(0.50-16.73)\end{array}$ & 0.84 & 1.25 & 2.45 \\
\hline DMP & $\begin{array}{c}0.001 \\
(\mathrm{ND}-69.17)\end{array}$ & 0.001 & 0.001 & 7.24 \\
\hline DMTP & $\begin{array}{c}5.33 \\
(\mathrm{ND}-24.24)\end{array}$ & 3.58 & 5.30 & 11.54 \\
\hline DMDTP & $\begin{array}{c}0.001 \\
(\mathrm{ND}-40.73)\end{array}$ & 0.001 & 0.001 & 1.21 \\
\hline$\sum$ DAP*** & $\begin{array}{c}153.82 \\
(64.69-476.60)\end{array}$ & 70.96 & 153.82 & 309.50 \\
\hline
\end{tabular}

*Los datos se reportan como mediana y entre paréntesis se ofrece las concentraciones mínima y máxima $(\mathrm{n}=29)$; **los percentiles fueron calculados con el paquete estadístico SPSS; $* * * \sum O C$ : sumatoria de la concentración urinaria de los seis metabolitos dialquilfosfatos (DAP), expresada como la mediana y entre paréntesis los valores mínimo y máximo.

DEP: dietilfosfato; DETP: dietiltiofosfato; DEDTP: dietilditiofosfato; DMP: dimetilfosfato; DMTP: dimetiltiofosfato; DMDTP: dimetilditiofosfato; ND: no detectable.

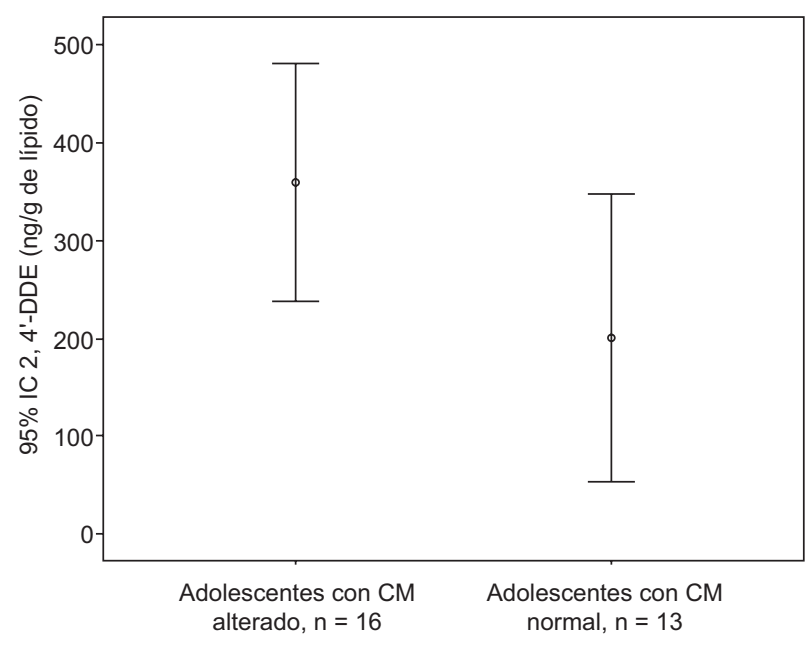

Fig. 3. Comparación entre las concentraciones plasmáticas del 2,4'-dicloro difenil dicloroetileno (2,4'-DDE) expresada como mediana e intervalo de confianza (IC) al $95 \%$, y el comportamiento del ciclo menstrual (CM). CM alterado: corto o largo. CM normal: 24 a 32 días. U de Mann-Whitney: $\mathrm{p}=0.03$. del perfil hormonal (Fig. 4) fue significativa únicamente cuando las jóvenes fueron agrupadas de acuerdo con el comportamiento de la hormona $\mathrm{LH}$ (alterado, no alterado y cursando con el periodo menstrual al momento de la toma de la muestra de sangre) (lambda de Wilks $=0.029, \mathrm{~F}_{(36,18)}=$ 2.45, $\mathrm{p}<0.02, \mathrm{n}=29$ ). El análisis generó dos funciones discriminantes: el grupo de adolescentes con niveles séricos alterados de LH se separó significativamente del grupo de participantes cuyos valores de esta hormona fueron normales (distancia de Mahalanobis $=124.50, p=0.10$; Fig. 4). En la misma figura se puede apreciar que las participantes que estaban cursando con su periodo menstrual al momento de la recolección de la muestra de sangre para el análisis del perfil hormonal, no resultaron significativamente separadas de los dos grupos (distancia de Mahalanobis $=51.77, \mathrm{p}=0.68$ ). En el cuadro III se enlistan los ocho plaguicidas que de manera significativa contribuyeron en la separación de estos grupos. 


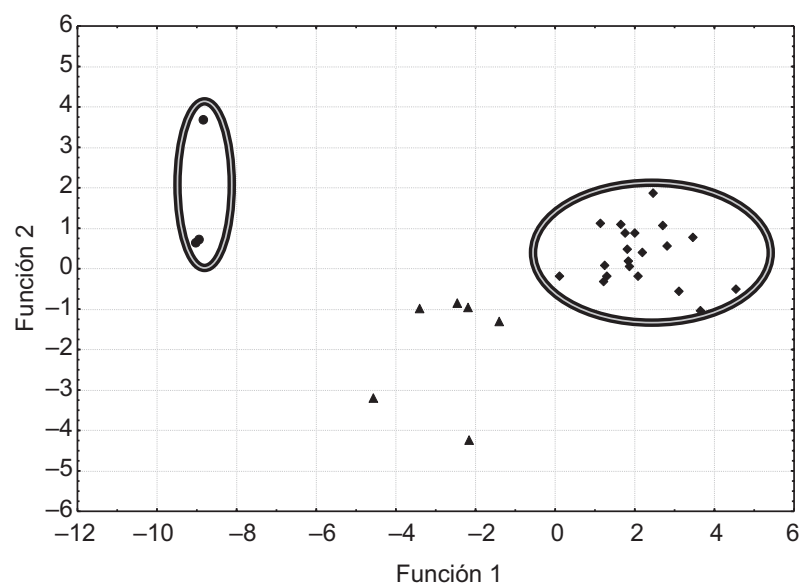

Fig. 4. Funciones canónicas del análisis discriminante de las concentraciones plasmáticas de plaguicidas organoclorados y urinarias de los metabolitos dialquilfosfatos de las adolescentes participantes, agrupadas de acuerdo con: alteraciones en la concentración sérica de la hormona $\mathrm{LH}$ $(n=4)$, sin alteraciones $(n=19)$, y quienes cursaban con su periodo menstrual $(\mathrm{n}=6)$ al momento de la recolección de la muestra sanguínea.

CUADRO III. PLAGUICIDAS QUE CONTRIBUYERON SIGNIFICATIVAMENTE EN LA SEPARACIÓN DE LOS TRES GRUPOS DEL ANÁLISIS DISCRIMINANTE.

\begin{tabular}{lccc}
\hline Plaguicida & $\begin{array}{c}\text { Lambda de } \\
\text { Wilks }\end{array}$ & $\begin{array}{c}\mathrm{F} \\
(\mathrm{n}=29)\end{array}$ & $\begin{array}{c}\text { Valor de } \\
\mathrm{p}\end{array}$ \\
\hline DMP & 0.05 & 4.74 & 0.03 \\
DEDTP & 0.06 & 5.61 & 0.02 \\
Hexaclorobenceno & 0.06 & 6.19 & 0.02 \\
Heptacloro epóxido & 0.06 & 5.68 & 0.02 \\
Trans-nonaclor & 0.08 & 8.71 & 0.007 \\
Cis-nonachor & 0.11 & 13.87 & 0.001 \\
2,4'-DDE & 0.07 & 7.16 & 0.01 \\
4,4'-DDE & 0.10 & 11.41 & 0.003 \\
\hline
\end{tabular}

DMP: dimetilfosfato; DEDTP: dietilditiofosfato; 2,4'-DDE : 2,4'-dicloro difenil dicloroetileno; 4,4'-DDE : 4,4'- dicloro difenil dicloroetileno; F: Fisher calculada; p: probabilidad.

\section{DISCUSIÓN Y CONCLUSIONES}

La exposición a estos plaguicidas tiene un importante impacto en la salud de las mujeres en edad reproductiva. Estudios reportados en la literatura han evidenciado que los contaminantes evaluados pueden causar problemas neurológicos, metabólicos, genéticos y reproductivos (Yáñez 2000, Carod 2002, Charboneau y Koger 2008, McMillen et al. 2008, Ramírez-Jiménez 2015).

En un estudio realizado por Yáñez et al. (2000) en el que se evaluó la exposición de mujeres a DDT en la Huasteca Potosina, se reportaron concentraciones plasmáticas de este insecticida y de sus metabolitos superiores a las detectadas en el presente trabajo ( DDT $1163 \mathrm{ng} / \mathrm{g}$ de lípidos vs $\Sigma$ DDT $601.23 \mathrm{ng} / \mathrm{g}$ de lípidos). Las casas habitación de ambas poblaciones están ubicadas en áreas endémicas de paludismo en las que se roció este insecticida por aproximadamente 60 años (ISAT 2001), por lo que ambas comunidades presentan una exposición crónica al DDT residual y a sus metabolitos. La diferencia entre las concentraciones detectadas en ambos estudios puede explicarse porque el estudio de Yáñez et al. (2000) se realizó cuando aún se empleaba el DDT para el control del paludismo, mientras que el presente estudio se desarrolló 14 años después de la última aplicación de este insecticida. En un estudio realizado por AlvaradoHernández et al. (2013) en mujeres que eran atendidas de parto en un hospital ubicado en la misma región donde se desarrolló el presente trabajo, los autores evaluaron la exposición materna a una mezcla de plaguicidas OC, obteniendo como resultado de la $\Sigma$ de OC una media de $10600 \mathrm{ng} / \mathrm{g}$ de lípidos, la cual es tres veces más alta que la concentración promedio detectada en el presente reporte de investigación (3653.44 ng/g de lípidos). En ambos estudios se monitorearon los mismos plaguicidas OC (aldrin, a$\mathrm{BHC}, \mathrm{b}-\mathrm{BHC}, \mathrm{g}-\mathrm{BHC}$, hexaclorobenceno, heptacloro epóxido, trans-nonaclor, cis-nonaclor, 4,4'-DDE y 4,4'-DDT), lo que corrobora la persistencia de estos compuestos químicos y por ende el riesgo de exposición crónica a residuos de estos contaminantes para quienes habitan en la zona.

Ramírez-Jiménez et al. (2014) evaluaron la exposición infantil a plaguicidas OF en El Refugio (ubicado en misma zona del presente estudio). Estos autores reportan al DMTP como el metabolito DAP más frecuente, con una concentración media en orina de $0.02 \mathrm{mg} / \mathrm{g}$ de creatinina. En nuestro estudio, este metabolito DAP también fue el más frecuente, pero la concentración media detectada fue notoriamente superior $(7.69 \mathrm{mg} / \mathrm{g}$ de creatinina).

Son escasos los estudios en mujeres no expuestas ocupacionalmente a agroquímicos que hayan empleado herramientas objetivas para valorar la relación de esta exposición tanto con los niveles de hormonas sexuales como con las características del CM, como es el caso del estudio aquí reportado, que se apoyó con el empleo de un diario/bitácora (diario menstrual), para registrar todos los eventos relacionados con el CM.

Chen et al. (2005) evaluaron la exposición a DDT en el comportamiento menstrual de mujeres migrantes de China a Estados Unidos. Los autores reportaron que aunque en el análisis univariado hubo una 
asociación entre los niveles de 4,4'-DDE y la duración del ciclo menstrual, no tuvieron suficiente evidencia para aseverar que el DDT o el DDE alteraron el CM o el periodo. Cabe señalar que el registro de las características del CM de las mujeres participantes se hizo con base en entrevistas, por lo que el resultado podría estar sesgado. En contraste, en el presente estudio se empleó un diario/bitácora (en el que las adolescentes anotaron durante 15 meses las características tanto del ciclo como del periodo menstrual) para la evaluación del comportamiento del CM.

Mclachlan et al. (2006) realizaron un estudio sobre aquellos compuestos exógenos que son considerados perturbadores endocrinos, tales como los plaguicidas OC. Estos autores reportaron que el DDE (principal metabolito del DDT) es un estrógeno ambiental, ya que imita al estrógeno endógeno, convirtiéndose en modulador endocrino que altera las concentraciones de estradiol y progesterona. Estos cambios hormonales sólo habían sido asociados con la exposición a altas concentraciones de DDE (10 ng/ $\mathrm{mL}$ ). Por otro lado, Windham et al. (2005) reportaron una asociación entre concentraciones altas de DDE (mediana de $13.1 \mathrm{ng} / \mathrm{mL}$ ) en una población de 48 mujeres campesinas, y la presencia de ciclos cortos y disminución de niveles séricos de progesterona. Este comportamiento también se observó en nuestra población de estudio, pero con un nivel de exposición 10 veces menor (mediana del 2,4'-DDE y 4,4'-DDE igual a 1.13 y $1.52 \mathrm{ng} / \mathrm{mL}$, respectivamente). Estos resultados se podrían explicar por la capacidad de este contaminante para inhibir a la enzima responsable de la síntesis de progesterona, de acuerdo con los reportes de Cooper et al. (2005).

En un estudio realizado por Germain et al. (2012), en el cual se evaluó la exposición a plaguicidas OC (hexaclorobenceno, aldrin, lindano, heptacloro y DDE, entre otros), y la duración de los ciclos menstruales de mujeres clínicamente sanas, se observó que a mayor concentración plasmática de estos plaguicidas las participantes presentaban con más frecuencia alteraciones en la duración de sus CM. Los autores concluyeron que esta alteración podría deberse a que los OC reducen la producción de hormonas sexuales. En el presente estudio, las adolescentes que reportaron $\mathrm{CM}$ cortos tuvieron concentraciones plasmáticas elevadas de aldrin, heptacloro, lindano, 2,4'-DDE y 4,4'-DDE, aunque no se logró demostrar una asociación estadísticamente significativa, a diferencia de las mujeres que están ocupacionalmente expuestas a agroquímicos (trabajadoras de huertos, invernaderos y floristas), quienes presentan una asociación con las alteraciones tanto del periodo como del $\mathrm{CM}$, presentando además sangrados intermenstruales, periodos de amenorrea, endometriosis, ovarios poliquísticos e infertilidad (Farr et al. 2004, Windham et al. 2005, Axmon 2006, Rasier et al. 2006, Germain et al. 2012, López 2013).

Tanto en el estudio realizado por Germain et al. (2011) como en éste, se tiene la certeza del comportamiento del $\mathrm{CM}$, ya que en ambos se empleó un diario/bitácora para registrar todos los eventos, con la diferencia de que los autores citados sólo lograron que 17 mujeres de las 83 participantes lo llevaran cabalmente durante siete ciclos, mientras que en nuestro estudio el total de adolescentes (29) completó el registro de 15 meses. En ambos trabajos queda por demás evidenciada la complejidad de este tipo de investigaciones.

Freire et al. (2013) realizaron un estudio en el cual evaluaron la asociación entre concentraciones de plaguicidas OC y alteraciones de las hormonas sexuales en mujeres pre, peri y postmenopáusicas, encontrando sólo asociaciones inversas entre LH y FSH y las concentraciones de HCB, DDT, DDE, mirex y aldrin en las mujeres peri y post menopaúsicas, no así en el grupo de mujeres premenopáusicas. Los autores concluyeron que los niveles elevados de prolactina detectados en su población de estudio sugieren un efecto estrogénico de estos contaminantes. Esto lleva a reflexionar en que la capacidad de los plaguicidas OC como perturbadores endocrinos es preocupante (Charboneau y Koger 2008), a pesar de que los resultados obtenidos en el presente trabajo no evidenciaron su efecto sobre el perfil hormonal, probablemente por el tamaño de muestra.

Nuestro estudio es el primer reporte en la literatura que evalúa el efecto de los plaguicidas OC sobre el perfil hormonal y el comportamiento del $\mathrm{CM}$ en adolescentes no expuestas ocupacionalmente a plaguicidas organofosforados. En el $50 \%$ de las adolescentes que presentaron alguna alteración en los niveles séricos de hormonas sexuales (LH, FSH, progesterona, estradiol o prolactina) se detectaron cuatro de los seis metabolitos DAP en orina, y todas tuvieron además concentraciones urinarias por arriba del LD $(0.69 \mathrm{ng} / \mathrm{mL})$ del metabolito DMTP. Respecto a la duración del CM, se observó que a mayor concentración en orina de $\Sigma$ DAP, la frecuencia de ciclos cortos ( $<24$ días) fue estadísticamente superior $(\mathrm{p}=$ 0.02 , datos no mostrados).

Los resultados obtenidos del análisis discriminante, con el que se demostró la influencia de la exposición a plaguicidas OF (presencia en orina de los metabolitos DMP y DEDTP) y OC (hexaclorobenceno, heptacloro epóxido, trans-nonaclor, cis-nonaclor, 2,4'-DDE y 4,4'-DDE) sobre los niveles séricos de 
$\mathrm{LH}$, concuerdan con lo reportado por Bapayeva et al. (2016), quienes demostraron que la exposición a plaguicidas OC (entre ellos lindano y DDT), afecta los niveles séricos de las hormonas sexuales, principalmente FSH y estradiol. Aunque dichos autores no reportaron un resultado significativo con relación a LH, sus datos evidencian que los agroquímicos actúan como perturbadores endocrinos.

Tomando esto como base, una posible explicación de los resultados obtenidos en nuestro estudio es que los compuestos OC (como el DDE y el g-BHC) afectan el perfil hormonal a nivel del ovario dada su demostrada capacidad estrogénica (Freire et al. 2013).

Guo et al. (2017) evaluaron a 187 mujeres con ovario poliquístico y la asociación de este trastorno con la exposición a plaguicidas OC, específicamente DDT y sus metabolitos y HCH. Dichos autores no encontraron diferencias estadísticamente significativas entre las concentraciones de los plaguicidas diclorodifenildicloroetano (DDD), DDE y $\mathrm{HCH}$ y los dos grupos participantes (pacientes con ovario poliquístico vs control sano), pero sí respecto a las concentraciones de DDT, ya que en mujeres con ovario poliquístico los niveles de este insecticida fueron mayores $(p=0.001)$. Los autores concluyeron que la exposición a este compuesto desempeña un papel importante en la patogénesis del síndrome como consecuencia de la alteración que genera en el perfil hormonal.

Greenlee et al. (2003) evaluaron los factores de riesgo de infertilidad femenina en una comunidad agrícola, y concluyeron que hay una relación estadísticamente significativa entre los casos de infertilidad y el uso de herbicidas (como algunos OF), no así con la exposición a fungicidas. Los autores sugieren no perder de vista otros factores como el tabaquismo y el consumo de alcohol, entre otros.

Una importante limitante de diversos estudios realizados en mujeres es que tanto la evaluación de la exposición a los agroquímicos como el registro del comportamiento del ciclo menstrual, se realizaron con base en la aplicación de cuestionarios sobre usos y costumbres, así como de entrevistas. De ahí la relevancia del presente trabajo, ya que para evaluar la magnitud de la exposición a agroquímicos se cuantificaron en plasma los plaguicidas OC y en orina los metabolitos de los OF, en tanto que todos los eventos relacionados con el CM se evidenciaron mediante el empleo del diario/bitácora. Este también permitió conocer con precisión el día en que cada una de las participantes se encontraba en su CM cuando fue analizado su perfil hormonal.
Este estudio difícilmente puede evidenciar las consecuencias a largo plazo de la exposición no ocupacional a agroquímicos sobre la salud reproductiva de las adolescentes; sin embargo, reportes en la literatura han señalado alteraciones severas tanto en las madres como en sus hijas, tales como cáncer de mama, infertilidad, endometriosis, ovarios poliquísticos, aumento de riesgo de presentar abortos espontáneos, inhibición de la producción hormonal, pubertad precoz, malformaciones en la formación de los folículos maduros, alteraciones en el desarrollo del producto de la gestación, bajo peso al nacer y deformaciones en los órganos reproductores, entre otras, a consecuencia de la exposición a estos contaminantes (Andrade et al. 2006, Uzumcu y Zachow 2007, ISTAS 2010, Bergman et al. 2012, Fowler et al. 2012, Germain et al. 2012).

La Academia Americana de Pediatría sugiere que no sólo las adolescentes sino también los padres de familia y el sector salud conozcan el comportamiento del CM y los factores de riesgo para prevenir afectaciones cuyas consecuencias podrían verse reflejadas en la edad adulta como infertilidad (AAP 2006).

El presente estudio muestra la complejidad del escenario de exposición no ocupacional a mezclas de plaguicidas en adolescentes de una comunidad agrícola; asimismo, los diferentes factores del ciclo menstrual que pueden verse afectados por esta exposición, como alteraciones de los niveles séricos de las hormonas sexuales, y la duración de los periodos y de los ciclos. Estos hallazgos deben considerarse como base para generar programas de educación ambiental y reproductiva cuya finalidad sea prevenir daños a largo plazo y a las siguientes generaciones, así como medidas de protección para las poblaciones que viven en estos escenarios.

Cabe señalar que una de las limitantes del presente trabajo fue el tamaño de la muestra, que probablemente ésta sea la causa de que la mayoría de las asociaciones entre la magnitud de la exposición a agroquímicos y el perfil hormonal y la duración del CM y del periodo, no fuesen significativas. No obstante, sí se observó una tendencia positiva (a mayor exposición a agroquímicos, mayor alteración del $\mathrm{CM}$ ), lo que implica una relevancia clínica, sobre todo para la salud reproductiva de las participantes. De ahí la importancia de continuar con esta línea de investigación, realizando estudios no sólo con un número mayor de participantes sino también con la inclusión de otros factores que pueden contribuir a las alteraciones tanto en el perfil hormonal como del periodo y el CM, tales como la calidad de la dieta, condiciones de vida (estrés), actividad sexual 
(uso de anticonceptivos), adicciones (alcoholismo, drogadicción y tabaquismo) y práctica de ejercicio, entre otros. Esto evidenciaría el impacto negativo de la exposición no ocupacional a agroquímicos en la salud reproductiva femenina.

\section{AGRADECIMIENTOS}

Gracias al apoyo del Consejo Nacional de Ciencia y Tecnología, que otorgó la beca con número 484878. El proyecto se realizó con el apoyo del Instituto de la Mujer de San Luis Potosí y fondos de la Comisión para la Cooperación Ambiental de Canadá y del Laboratorio de Género, Salud y Ambiente de la Facultad de Medicina de la Universidad Autónoma de San Luis Potosí.

\section{REFERENCIAS}

AAP (2006). Menstruation in girls and adolescents: Using the menstrual cycle as a vital. Pediatrics 188 (5), 2245-2250. https://doi.org/10.1542/peds.2006-2481

Alvarado-Hernández D.L., Montero-Montoya R., SerranoGarcía L., Arellano-Aguilar O., Jasso-Pineda Y. y Yáñez-Estrada L. (2013). Assessment of exposure to organochlorine pesticides and levels of DNA damage in mother-infant pairs of an agrarian community. Environ. Mol. Mut. 54 (2), 99-111. https://doi.org/10.1002/ em.21753

Andrade A., Pacheco A., Nóbrega C. y Mendes A. (2006). Disruptores endocrinos: potencial problema para la salud y medio ambiente. Biomed. 17 (2), 146-150.

AOAC (2015). Official methods of analysis of AOAC international. Association of Analitycal Chemistry. Guía de validación. Rockville, EUA, 38 pp.

Axmon A. (2006). Menarche in women with high exposure to persistent organochlorine pollutants in utero and during childhood. Environ. Res. 102 (1), 77-82. https:// doi.org/10.1016/j.envres. 2005.12.014

Bapayeba G., Issayeva R., Zhumadilova A., Nurkasimova R., Kulbayeva S. y Tleuzhan R. (2016). Organochlorine pesticides and female puberty in South Kazakhstan. Reprod. Toxicol. 65, 65-75. https://doi. org/10.1016/j.reprotox.2016.06.017

Bergman Å., Heindel J.J., Jobling S., Kidd K.A. y Zoeller R.T. (2012). Endocrine disrupting chemicals. United Nations Environment Programme-World Health Organization. Ginebra, 289 pp.

Bhatt R.V. (2000). Environmental influence on reproductive health. Int. J. Gynecol. Obstet. 70 (1), 69-75. https://doi.org/10.1016/s0020-7292(00)00221-6
Bretveld R.W., Thomas C.M., Scheepers P.T., Zielhuis G.A. y Roeleveld N. (2006). Pesticide exposure: The hormonal function of the female reproductive system disrupted. Reprod. Biol. Endocrinol. 4 (30), 1-14. https://doi.org/10.1186/1477-7827-4-30

Carod E. (2002). Insecticidas organofosforados. "De la guerra química al riesgo laboral y doméstico". Medifam 12 (5), 333-340.

CDC (2015). Índice y masa corporal para niños y adolescentes. Centro para el Control y Prevención de Enfermedades [en línea]. https://www.cdc.gov/ healthyweight/spanish/assessing/bmi/childrens_bmi/ acerca_indice_masa_corporal_ninos_adolescentes. html 26/12/2019

Charboneau J. y Koger S. (2008). Plastics, pesticides and PBDEs: Endocrine disruption. J. Dev. Phys. Disabil. 20 (2), 115-128. https://doi.org/10.1007/s10882-0079083-3

Chen A., Jun Z., Lifeng Z., Gao E., Chen L., Rogan W. y Wolff M. (2005). DDT serum concentration and menstruation among young Chinese women. Environ. Res. 99, 397-402. https://doi.org/10.1016/j. envres.2004.12.015

Cooper G., Klebanoff M., Promislow J., Brock J. y Longnecker M. (2005). Polychlorinated biphenyls and menstrual cycle characteristics. Epidemiology 16 (2), 191-200. https://doi.org/10.1097/01. ede.0000152913.12393.86

Escobar M.E, Pipman V., Arcari A., Boulgourdjian E., Keselman A., Pasqualini T., Alonso G. y Blanco M. (2010). Trastornos del ciclo menstrual en la adolescencia. Arch. Argent. Pediatr. 108, 363-369.

Farr S.L., Cooper G.S., Cai J., Savitz D.A. y Sandler D.P. (2004). Pesticide use and menstrual cycle characteristics among premenopausal women in the agricultural. Am. J. Epidemiol. 160 (12), 1194-1204. https://doi. org/10.1093/aje/kwi006

Foradori C.D., Hinds L.R., Quihuis A.M., Lacagnina A.F., Breckenridge C.B. y Handa R.J. (2011). The differential effect of atrazine on luteinizing hormone release in adrenalectomized adult female Wistar rats. Biol. Reprod. 85, 684-689. https://doi.org/10.1095/ biolreprod.111.092452

Fowler P.A.F., Bellingham M., Sinclair K.D., Evans N.P., Pocar P., Fischer B. y O'Shaughnessy P. J. (2012). Impact of endocrine-disrupting compounds (EDCs) on female reproductive health. Mol. Cell. Endocrinol. 355(2), 231-239. https://doi.org/10.1016/j. mce.2011.10.021

Freire C., Koifman R.J., Novaes P., Sarcinelli P.N., Rosa A.C., Clapauch R. y Koifman S. (2013). Association between serum levels of organochlorine pesticides and sex hormones in adults living in heavily contaminated 
area in Brazil. Int. J. Hyg. Environ. Health. 217, 370378. https://doi.org/10.1016/j.ijheh.2013.07.012

Germaine M.L., Rios L., McLein M., Cooney M., Kostyniak P. y Sundaram R. (2011). Persistent organochlorine pollutants and menstrual cycle characteristics. Chemosphere 85(11), 1742-1748. https://doi.org/10.1016/j. chemosphere.2011.09.027

Germaine M.L., Chen Z., Peterson C.M., Hediger M.L., Croughan M.S., Sundaram R., Stanford J.B., Varner M.W., Fujimoto V.Y., Giudice L.C., Trumble A., Parsons P.J., y Kannan K. (2012). Persistent lipophilic environmental chemicals and endometriosis: The ENDO Study. Environ. Health. Perspect. 120 (6), 811816. https://doi.org/10.1289/ehp.1104432

Greenlee A., Arbuckle T. y Chyou P. (2003). Risk factors for female infertility in an agricultural region. Epidemiology 14 (4), 429-436. https://doi.org/10.1097/01. EDE.0000071407.15670.aa

Guo Z., Qiu H., Wang L., Wang C., Chen M. y Zuo Z. (2017). Association of serum organochlorine pesticides concentrations with reproductive hormone levels and polycystic ovary syndrome in a Chinese population. Chemosphere 171, 595-600. https://doi.org/10.1016/j. chemosphere.2016.12.127

Hovander L., Athanasiadou M., Asplund L., Jensen S. y Klasson E. (2000). Extraction and cleanup methods for analysis of phenolic and neutral organohalogens in plasma. J. Anal. Toxicol. 24, 696-703. https://doi. org/10.1093/jat/24.8.696

INAFED-SEGOB (2010). Enciclopedia de los municipios y delegaciones de México. Estado de San Luis Potosí. Instituto para el Federalismo y el Desarrollo Municipal-Secretaría de Gobernación. [en línea]. http://siglo. inafed.gob.mx/enciclopedia/EMM24sanluispotosi/ index.html 26/12/2019

ISAT (2001). Diagnóstico situacional del uso de DDT y el control de la malaria. Instituto de Salud, Ambiente y Trabajo de México. Informe Regional para México y Centroamérica. Ciudad de México, México, 58 pp.

ISTAS (2010). Disruptores endocrinos: un nuevo riesgo tóxico. Instituto Sindical de Trabajo, Ambiente y Salud. Folleto de información. Valencia, España, 10 pp.

Levario M., Amato D., Ostrosky P., González C., Corona Y. y Sanin L. (2004). Relation between pesticide exposure and intrauterine growth retardation. Chemosphere 55 (10), 1421-1427. https://doi.org/10.1016/j.chemosphere.2003.11.027

López G. (2013). Eventos reproductivos adversos en parejas de trabajadores agrícolas de Muna, Yucatán. Tesis de Licenciatura. Facultad de Medicina, Universidad Autónoma de Yucatán, Mérida, México, 130 pp.

Mclachlan J., Simpson E. y Martin M. (2006). Endocrine disrupters and female reproductive health. Best. Pract.
Res. Clin. Endocrinol. Metab. 20 (1), 63-75. https:// doi.org/10.1016/j.beem.2005.09.009

McMillen I.C., MacLaughlin S.M., Muhlhausler B.S., Gentili S., Duffield J.L. y Morrison J.L. (2008). Developmental origins of adult health and disease: The role of periconceptional and fetal nutrition. Basic Clin. Pharmacol. Toxicol. 102 (2), 82-89. https://doi. org/10.1111/j.1742-7843.2007. 00188.x

Melmed S., Polonsky K., Larsen P. y Kronenberg H. (2017). Williams. Tratado de endocrinología. 13a ed. Elsevier, Barcelona, España, 1936 pp.

Nicolopou P. y Pitsos M.A. (2001). The impact of endocrine disrupters on the female reproductive system. Hum. Reprod. Update. 7 (3), 323-330. https://doi. org/10.1093/humupd/7.3.323

Ouyang F., Perry M.J., Venners S.A., Chen C., Wang B., Yang F., Fang Z., Zang T., Wang L., Xu X. y Wang Xu. (2005). Serum DDT, age menarque and abnormal menstrual cycle. Occup. Environ. Med. 62 (12), 878884. https://doi.org/10.1136/oem.2005.020248

Ramírez-Jiménez R., Mejía R., Calderón J., Montero R. y Yáñez L. (2014). Concentraciones urinarias de metabolitos de plaguicidas organofosforados en niños y adolescentes de una zona agrícola de San Luis Potosí. Revista Iberoamericana de Ciencias 1 (4), 87-97 pp.

Ramírez-Jiménez R. (2015). Asociación entre la exposición a plaguicidas OF's y la paraxonasa 1 (PON1) $y$ alteraciones neurocognitivas en niños y adolescentes de una comunidad agrícola de San Luis Potosí. Tesis de Doctorado. Facultad de Medicina, Universidad Autónoma de San Luis Potosí, San Luis Potosí, México, 153 pp.

Rasier G.T., Toppari J., Parent A.S. y Bourguigno J.P. (2006). Female sexual maturation and reproduction after prepubertal exposure to estrogens and endocrine disrupting chemicals: a review of rodent and human data. Mol. Cell. Endocrinol. 254, 187-201. https://doi. org/10.1016/j.mce.2006.04.002

Rattner B.A., Sileo L. y Scanes C.G. (1982). Oviposition and the plasma concentrations of $\mathrm{LH}$, progesterone and corticosterone in bobwhite quail (Colinus virginianus) fed parathion. J. Reprod. Fert. 66 (1), 147-155. https:// doi.org/10.1530/jrf.0.0660147

Rivero N. (2012). Evaluación de los efectos en salud por la exposición a plaguicidas en niños de San Luis Potosí. Tesis de Doctorado. Facultad de Medicina, Universidad Autónoma de San Luis Potosí, San Luis Potosí, México, 179 pp.

Roselli M., Reinhart K., Imthurn B., Keller P.J. y Dubey R. (2000). Cellular and biochemical mechanism by which environmental oestrogens influence reproductive function. Hum. Reprod. Update. 6 (4), 332-350. https://doi.org/10.1093/humupd/6.4.332 
Roselló M.A., Morales F., Pimentel D., Abadi T., Bustos H.H. y Casanueva E. (2003). Ciclo menstrual y alimentación. Perinatol. Reprod. Hum. 17 (2), 61-71.

SPSS (2009). PASW statistics for Windows, version 18.0. SPSS, Chicago.

StatSoft (2007). Statistica base (programa informático) [en línea]. http://www.statsoft.com 26/12/2019

Tabachnick B.G. y Fidell L.S. (2007). Using multivariate statistics. 7a ed. Pearson Education, Nueva York, EUA, 815 pp.

Tiemann U. (2008). In vivo and in vitro effects of the organochlorine pesticides DDT, TCPM, methoxychlor, and lindane on the female reproductive tract of mammals: A review. Reprod. Toxicol. 25 (3), 316-326. https://doi.org/10.1016/j.reprotox.2008.03.002

Uzumcu M. y Zachow R. (2007). Developmental exposure to environmental endocrine disruptors: Consequences within the ovary and on female reproductive function. Reprod. Toxicol. 23 (3), 337-352. https://doi. org/10.1016/j.reprotox.2006.10.006

Valcke M., Samuel O., Bouchard M., Dumas P., Belleville D. y Tremblay C. (2006). Biological monitoring of exposure to organophosphate pesticides in children living in peri-urban areas of the Province of Quebec, Canada. Int. Arch. Occup. Environ. Health. 79 (7), 568-577. https://doi.org/10.1007/s00420-006-0085-8
Windham G., Lee D., Mitchell P., Anderson M., Petreas M. y Lasley B. (2005). Exposure of organochlorine compounds and effect on ovarian function. Epidemiology 16 (2), 182-190. https://doi.org/10.1097/01. ede.0000152527.24339.17

Wojtys E.M., Jannausch M.L., Kreinbrink J.L., Harlow S.D. y Sowers M.R. (2014). Athletic activity and hormone concentrations in high school female athletes. J. Athl. Train. 50 (2), 185-192. https://doi. org/10.4085/1062-6050-49.3.62

Yáñez L. (2000). Efecto genotóxico del DDT en comunidades indígenas con paludismo endémico. Tesis de Doctorado. Facultad de Medicina, Universidad Autónoma de San Luis Potosí, San Luis Potosí, México, $65 \mathrm{pp}$.

Yáñez L., Jiménez R., Athanasiadou M., Mejía R. y López D. (2000). Evaluación de la exposición perinatal al DDT y sus metabolitos en mujeres mexicanas. En: Género, ambiente y contaminación por substancias químicas (del Pont R., Ed.). SEMARNAT, Ciudad de México, México, pp. 115-132.

Zellnera D.A., Garriga A., Centeno S. y Wadsworth E. (2004). Chocolate craving and the menstrual cycle. Appetite 42 (1), 119-121. https://doi.org/10.1016/j. appet.2003.11.004 